\title{
新时代之下如何打破女性就业困境一一基于劳动力市场性别困 境视角的分析
}

\author{
李慧芬 \\ 南昌大学公共管理学院 \\ DOI:10.32629/ej.v3i3.470
}

\begin{abstract}
[摘 要] 在社会经济与文明不断发展的今天, 企业女性人才教育程度和社会地位逐渐提高,女性公平就 业的问题也日益成为人们关注的焦点, 就业是女性获得经济独立与财务自由的重要途径, 然而当前社会, 女性就业正面临着许多挑战,遭受着许多不公平的待遇, 因此, 如何打破求职者就业的性别困境, 促进社会 就业公平化,是我们需要审慎思考的一个问题。本文从劳动力市场性别困境视角切入,从现状、成因等层 面入手,分析了当今时代女性的就业困境,并就如何破解性别对女性就业的束缚,打破性别差别桎梏,促进 就业公平提出了相应对策, 旨在为改善新时代女性就业 “生态环境”, 使女性更好地实现自身的人力资本 的价值提供一些思路。
\end{abstract}

[关键词] 女性就业; 性别困境; 现状剖析; 对策建议

\section{引言}

近日,有一段有关打破女性就业困 境, 促进就业公平的公益广告在网络爆 红, 引发了许多女性劳动者的共鸣。在这 段广告中, 一幢偌大的写字楼里, 某公司 正在进行人员招聘, 公司的HR对前来应 聘的十几位男性应聘者提出了以下的问 题: 谈恋爱了吗?结婚了吗?打算什么时 候结婚呀?未来三年内打算生孩子吗?生 完孩子几个月之内能重返工作岗位上班? 打算生二胎吗?

一系列问题把前来面试的男性应聘 者问地满头雾水, 甚至有好几位应聘者 带着一脸的怀疑和满腔的愤怒转身离去, 他们大多认为这家公司的HR有些无理取 闹, 男生怎么会考虑生孩子、坐月子的事 情呢?的确, 这些问题对于男性应聘者而 言, 确实是有些匪夷所思。然而, 这些看 似匪夷所思的问题, 却真真切切地发生 在许多女性应聘者身上。

职场充满挑战, 职场人都或多或少 遇到过不公平的对待。根据智联招聘 《2020中国女性职场现状调查报告》显 示, 有 $58.25 \%$ 的女性遭遇了“应聘过程中 被问及婚姻生育状况”, 有 $27 \%$ 的女性遭
遇了“求职时, 用人单位限制岗位性别”, 有6.39\%的女性曾遭遇“婚育阶段被调岗 或降薪”, 而遭遇这些不公的男性屈指可 数。

由此可见, 女性求职遭遇的困境和 差别对待是一个广泛存在的问题。虽然 近几年国家也出台了相关的法律法规力 求保障女性在社会生活中平等的权利, 如我国《宪法》第四十八条规定: “中华 人民共和国的女性在社会生活的各个方 面与男子享有平等的权利。但事实上， 在人们现实的日常生活中, 女性就业依 然会遭遇来自各方面的阻力, 女性就业 性别困境问题广泛存在, 且大多数是以 隐性形式存在的, 女性就业困境日益加 深的趋势, 急需寻求有关解决措施来应 对此问题”。 \section{现状剖析}

1 我国求职者就业性别困境的

1. 1 什么是求职者就业性别困境?

针对这一定义性问题, 通过查阅相 关资料, 可以得知: 劳动力市场歧视, 是 指具有同等能力, 接受相同教育、培训和 相似经历并最终表现出相等的劳动生产 率的劳动者, 由于个人的本身特征所引
起的在就业、职业选择、升职、工资待 遇、培训等方面受到的不公平待遇。而 于女性求职者而言, 就业性别困境是指 女性在就业过程中遭受到的一系列不平 等对待, 包括用人单位提出的与职位无 关的, 有关性别婚姻状况、生育情况等问 题, 以及对女性提出高于男性的要求或 者同工不同酬等现象。引言中提到的广 告, HR对应聘者所问的有关 “结婚”、“生 育”、“是否要二胎” 等问题, 明显是一种 对女性求职者的就业性别差别对待。

1.2 当下我国女性求职者在就业过 程中遭遇的困境主要有哪些表现?

\subsection{1 就业机会不平等}

就业机会不平等主要体现在两个方 面。一方面, 部分用人单位的某些岗位在 招聘宣传上明确表示不招收女性求职者, 或者是虽然未明确表示不招收女性求职 者, 但是在实际面试过程中以各种不合 理、不相关的缘由淘汰女性求职者。

而另一方面是招聘录用标准不平等, 对女性面试者单方面拉高标准, 进行人 为设限, 淘汰女性求职者。部分用单位会 在相同的工作岗位、相同的工作环境下, 仅仅因为劳动者的性别差异而随心所欲 
地提高录用人才的标准, 对部分业务能 力强, 工作技能水平较高的女性求职者 形成隐性地压制。据相关数据显示, 现在 许多用人单位在招聘女性求职者时往往 会制定一些附加条件, 比如说: 身高、婚 姻、家庭、生育情况等。更为严重的是, 部分用人单位会要求女性就业者在刚上 岗投入工作的几年年内不允许生育, 而 其给出的解释却只是因为担心生育子女 可能会导致人才流失、教育及对孩子的 抚养和照顾会影响企业该岗位工作, 降 低生产效率, 导致用人单位人力资源成 本支出增加。

\subsection{2男女同工不同酬}

在我国, 女性的经济收入在量上虽 然较过去实现了较大幅度增长, 但相对 高收入人群仍然主要集中在男性, 在职 场中, 女性想要获得与男性同样的报酬 往往要为之付出多几倍的努力。

我国很多单位迫于社会或法律压力 招聘了女性员工, 但是在具体的操作之 中, 可能就会根据性别来划分差别, 即在 同等工作下, 给予女性职员较男性职员 更低的工资。

1.2. 3 就业待遇不平等 (尤其表现在 职业晋升受限制这一方面）

在许多的企业内部, 都有着这样的 “潜规则”, 若有某个岗位空闲, 公司内 部有两位员工 (一位是男性, 另一位是女 性) 有资格参与竞争, 但是在实际选拔的 过程中, 若两者能力相差无几, 公司高层 往往会优先选择男性候选人, 除非在女 性候选人的能力远超于男性候选人,才 有参与 “同台竞技” 的可能性。就算达 到此种可能性, 女性的晋升空间也远远 小于男性, 很容易便触及了所谓的职业 生涯 “天花板”, 女性的职业发展道路, 面临着极大的挑战。

\section{2 我国求职者就业性别困境的 原因分析}

我国求职者面临的就业性别困境, 从来都不是单方面的因素, 这与社会观 念、企业倾向、国家政策、人性等各方 面因素密切相关。本文主要从以下几点 进行分析:

2.1男女求职者在体力、生育等客观
生理条件上的差别

在这一点上, 我们不可否认, 在体力 方面的确大多数女性都不如男性, 因此 就无法胜任某些工作, 例如在某些需要 力气大或者体力强的工作, 很多女性求 职者都无法完成, 这在很大程度上压缩 了女性的求职空间, 女性不得不去一个 相对更狭小的领域和男性求职者竞争, 而现实是大多数工作都有相应的体力等 能力的要求, 这在无形之中就又大了竞 争, 使得女性就业陷入了一个求职“难上 加难”的困境, 遭遇着重重阻力。

2.2 女性自身综合素质欠缺, 难以胜 任岗位要求

由于长期的历史性因素的存在, 受 到传统重男轻女思想的影响, 女性接受 高中以上教育的人数少于男性, 导致女 性在学历方面处于劣势。女性就业观念 狭险, 对工作所处的地域环境、薪资待 遇、福利水平、出差频次、加班等要求 高, 对于工作单位的选择更倾向于机关 事业单位工作。诸多原因造成了女性自 身素质不高, 职场适应性差, 技能水平低, 难以胜任岗位的需要。

\section{3传统社会观念的影响}

从早先的奴隶社会时期开始, 中国 女性就饱受封建礼教观念的束缚, 在传 统的社会观念之下, 女性地位一直低于 男性, 社会文化要求女性要更多地服从 男性的安排, “三纲五常”、“男尊女卑” 的思想贯穿整个封建社会。

虽然随着时代的发展, 女性力量逐 渐崛起, 国家开始运用法律的手段出台 各种保护女性的规定, 女性地位一步步 提高, 但是依然存在着对女性的歧视与 偏见, 认为女性在体力、智力等各方面都 不如男性。

\section{4 企业追求利益最大化的倾向}

为了追求利润最大化, 用人者会想 方设法降低用人成本, 然而招用女工, 会 面临生育产假等各种支出, 这无疑会增 加用人成本、从而减少企业利润。所以, 为了追求更大利润, 很多企业在招聘时 就制定许多带有偏见性的规定。

2.5国家相关法律体系的不完善 根据相关事实表明, 目前我国并无
现行对性别歧视的明确界定, 也没有使 法官判决企业性别困境事例成立的现 行绝对标准。法律的缺失就造成法官虽 怜悯被歧视女性, 但也更多的还是充当 “劝和者” 的身份, 提供一个较为正式 和权威的平台供双方协调矛盾, 但这种 情况往往都是调解无果, 并且还伴随着 附加的风险, 即女性劳动者因这些合理 维权的行为激怒企业管理者而惨遭到 企业辞退。

3 改善女性就业生态,打破求 职者就业性别困境的对策建议

3.1女性求职者要提高自身综合素 质, 充分挖掘自身潜能, 提升就业竞争力

首先, 女性求职者要积极构建自己 的知识结构, 尽可能多地参加各种实践 活动, 锻炼个人能力。同时还要拓展社会 视野, 增加自我附加价值, 以提高个人整 体素质, 打造自身的不可替代性, 为自己 的职业生涯增添有效保障。其次要善于 发现自己的长处, 充分挖掘自身潜力, 排 除自身观念上的负性思维, 抓住机遇, 迎 接就业市场的挑战。

3. 2 转变传统社会思想, 树立性别平 等观念

现代社会的今天, 要加快转变传统 的社会思想, 树立男女性别平等的观念。 要发挥大众媒体的作用, 宣传男女平等 的观念意识, 以及与就业平等相关的法 律政策, 增强女性的自立意识与法律意 识, 并能够运用法律维护自身的权益。

3. 3 加大对有就业性别歧视现象存 在的企业的惩罚力度

提高企业在招聘过程中有偏见性招 人做法的违规成本。违规成本的上升可 以较为有效地降低用人单位的违规行为, 因为大多数的企业管理者都是出于理性 经济人的视角, 出于对降低企业经营风 险, 减少因违规带来的法律制裁成本。

3. 4健全法律法规系统, 保障女性合 法权利

法律上是保障人们权益是的最后一 道防线, 要想缓解女性求职的性别困境, 必须要建立健全相关的法律法规, 如借鉴 发达国家应对就业歧视的成功经验, 制定 专门的针对女性性别困境的法律, 并对 


\title{
解析事业单位档案信息安全管理问题
}

\author{
林秋利 \\ 吉林省洮南市档案馆 \\ DOI:10.32629/ej.v3i3.461
}

\begin{abstract}
[摘 要] 基于信息化时代背景下, 加快了事业单位档案管理信息化建设步伐, 切实提升了档案管理工作 水平与质量, 但也暴露出了许多安全性问题。本文简单分析了事业单位档案信息安全管理存在的主要问 题, 并结合具体问题提出了有针对性的解决措施。
\end{abstract}

[关键词] 事业单位; 档案管理; 信息安全; 问题; 解决措施

\section{1 事业单位档案信息安全管理 存在的主要问题}

1. 1 物理安全问题

从档案信息安全管理方面分析, 物 理安全指的是档案存放所处的外部环境 安全性问题, 比如存放的建筑环境与自 然环境。现阶段, 事业单位基本都是应用 电子档案管理方式, 其存在的物理安全 问题基本包含计算机软件、硬件设备问 题, 以及档案存放环境的温度、防火与防 盗等相关问题。比如, 部分计算机软件存 在安全漏洞被病毒侵入, 从而导致档案 资料无法应用或是被盗取; 部分计算机 硬件故障, 造成档案难以存储与读取; 外 部环境温度与湿度等可能会影响电子设 备的正常运行。

\section{2 人为安全问题}

档案管理工作是由人来完成的, 所 以人为因素也直接关系到事业单位档案
信息安全。纵观我国事业单位的档案管 理工作现状, 其中人为安全问题主要表 现如下: 管理专业人员贵乏, 尤其是电子 档案管理人员; 责任意识薄弱, 综合素质 水平比较低; 实践工作中忽略了档案信 息安全管理, 甚至因为错误操作导致档 案信息误删; 部分档案管理工作人员的 档案信息保密意识不足, 导致档案信息 外泄等。

\section{3技术安全问题}

事业单位档案管理引入计算机技术, 创新了档案管理方式, 提升了档案管理 效率与水平, 但也增加了档案信息安全 风险。在电子档案管理中应用计算机软 件, 需要连接外部设备, 且网络环境下很 容易遭受病毒入侵与黑客攻击, 同时计 算机软件运行与更新也可能会出现安全 漏洞, 特别是安全等级比较低的计算机 设备。除此之外, 技术安全问题也表现在
档案资料信息没有及时进行备份, 由于 计算机的存储量相对比较大, 若是计算 机发生了故障, 很可能会造成档案资料 信息无法恢复。

2 事业单位档案信息安全问题 的有效解决措施

针对事业单位的档案管理工作而言, 必须把信息安全管理作为根本性目标, 然后结合档案信息安全具体问题, 制定 有针对性、有目标性的解决措施。

2.1加强事业单位档案信息安全物 理防护

事业单位在进行档案信息安全物理 防护时, 需要高度重视档案室与档案存 放设备的物理防护。其中档案室应该进 行特殊设计, 展开全方面的安全评估, 基 本包含档案室温度、防火以及防盗等方 面, 然后结合具体需求配置齐全的档案 管理设备, 充分发挥档案管理作用, 切实
“性别偏差”、“隐性歧视” 等相关词语 做出明确的概念界定, 规定用人单位所 应承担的责任与违反后应负的后果, 加 快出台《反就业歧视法》。

\section{4 未来展望}

随着时代和社会经济的发展, 人 们的观念也会一步步转变, 逐渐摆脱 传统的 “男主外, 女主内” 等观念的桎 梏, 促进劳动力市场的公平性, 改善女 性就业的 “生态环境”, 使得女性求职 者能够更好地实现自身的人力资本价 值, 我期待着, 一个更友好、更健康时
代的到来。

\section{[参考文献]}

[1]石雷.女性就业歧视的原因及对策 分析[J].中国集体经济,2019,(7):155-157.

[2]刘睿.我国女性就业歧视问题的对策 研究[J].劳动保障世界,2018,(21):14-15.

[3]许荣伟.城市化视角下我国女性 就业歧视问题探究[J]. 福建论坛(人文社 会科学版),2012,(S1):119-120.

[4]葛玉好, 邓佳盟,张帅.大学生 就业存在性别歧视吗? - - 基于虚 拟配对简历的方法 [J]. 经济学(季
刊),2018,17(04):1289-1304.

[5]刘红,苗艺璇.女性就业歧视问题 研究 [J]. 商业经济,2014,(12):76-77.

[6]马荣荣.对中国女性就业中存在 的性别歧视问题的探讨一基于西方经 济学视角的分析 [J]. 东方企业文 化,2011,(18):226-227.

\section{作者简介:}

李慧芬(1999--), 女, 汉族, 江西贑州 人,本科在读,南昌大学公共管理学院,研 究方向：人力资源管理专业。 